\title{
Biofilme dental em crianças com erosão dentária
}

\author{
Dental biofilm in children with dental erosion
}

\author{
Caleb Shitsuka* \\ Maria Salete Nahás Pires Corrêa** \\ Danilo Antônio Duarte ${ }^{* * *}$ \\ Mariana Ferreira Leite ${ }^{* * * * *}$
}

\section{Resumo}

Objetivo: o presente estudo quantificou o biofilme dental por meio do índice de higiene oral simplificado (IHO-S) de Greene e Vermillion, em crianças com erosão dentária, comparando-as com crianças sem esse problema de saúde bucal. Sujeitos e método: participaram do estudo quarenta crianças, de quatro a nove anos de idade, examinadas por um único examinador treinado de acordo com o índice de O’Brien para o diagnóstico de erosão dentária, sendo que vinte crianças apresentavam erosão nos dentes e vinte crianças não a apresentavam. Posteriormente, elas receberam orientações e padronização para a avaliação do IHO-S. Os dados foram analisados pela regressão logística univariada de Poisson. Valores de razão de prevalência $(R P)$ e intervalos de confiança de 95\% (IC 95\%) foram calculados. Resultados: a média (desvio padrão) do IHO-S de crianças com erosão foi de 1,20 (0,40), menor que as crianças do grupo controle $1,75(0,45)$. A quantidade de biofilme foi associada à presença de erosão dentária (RPIC 95\% =0.390; 0.180-0.833) e valor de $p=0.027$ Conclusões: crianças com erosão nos dentes apresentaram uma quantidade significativamente menor de biofilme em relação às crianças sem esse problema. $O$ biofilme pode ser um possível fator protetor contra os ataques ácidos e o desenvolvimento da erosão dentária.

Palavras-chave: Erosão dentária. Biofilme dental. Placa dental.

\section{Introdução}

Considerada um problema de saúde bucal ${ }^{1}$, a erosão dentária é estudada há séculos ${ }^{2,3}$, porém, nos últimos anos, foi observado que a sua prevalência está aumentando, o que, devido às recentes mudanças no estilo de vida ${ }^{8,9}$, a torna um dos principais problemas de saúde bucal, principalmente em crianças e adolescentes ${ }^{4-7}$.

A erosão dentária é ocasionada por um processo químico de perda irreversível da estrutura mineral e superficial dos dentes, decorrente de uma agressão ácida sem o envolvimento bacteriano ${ }^{10,11}$. Sua etiologia é complexa e multifatorial, na qual os ácidos que chegam à cavidade bucal podem ser de origem intrínseca ou extrínseca ${ }^{12-14}$.

As lesões erosivas decorrentes da agressão ácida afetam algumas regiões específicas dos dentes, porém, as porções de esmalte localizadas próximas à margem gengival permanecem intactas, possivelmente devido ao biofilme acumulado na região que pode agir como uma barreira mecânica contra a agressão ácida e possivelmente ser um fator de proteçãa $0^{5,11,13,15}$.

Tanto a cárie como as doenças periodontais têm relação direta com o biofilme, pois alterações nestas microcolônias de bactérias, unidas por uma matriz e aderidas nas superfícies dentais, podem modifi-

Mestre em Odontopediatria e doutorando em Ciências Odontológicas, Departamento de Ortodontia e Odontopediatria, Faculdade de Odontologia da Universidade de São Paulo, São Paulo, SP, Brasil

Mestre e doutora em Odontopediatria, professora livre docente, Departamento de Ortodontia e Odontopediatria, Faculdade de Odontologia da Universidade de São Paulo, São Paulo, SP, Brasil

Mestre e doutor em Odontopediatria, professor, Departamento de Odontopediatria, Faculdade de Odontologia, Universidade Cruzeiro do Sul, São Paulo, SP, Brasil.

Mestre e doutora em Materiais Dentários, professora titular, Departamento de Odontopediatria, Faculdade de Odontologia da Universidade Cruzeiro do Sul, São Paulo, SP, Brasil. 
car toda a condição de saúde, transformando-se em doença ${ }^{16}$. Dessa forma, também pode haver uma relação com a erosão dentária.

Apesar de alguns autores apontarem o biofilme dental como sendo possivelmente um fator de proteção contra os desafios ácidos, faltam estudos relacionando o potencial protetor do biofilme com erosão dentária. O presente estudo teve como objetivo quantificar a presença do biofilme dental nas superfícies dos dentes em crianças com erosão dentária, comparando-as com crianças sem a doença, para estabelecer uma possível relação do biofilme com a erosão dentária.

\section{Sujeitos e método}

A pesquisa foi aprovada pelo Comitê de Ética em Pesquisa da Universidade Cruzeiro do Sul, sob protocolo de número 015/2010.

Realizou-se um estudo transversal, com uma amostra de quarenta crianças, na faixa etária entre quatro e nove anos de idade, de ambos os sexos, que frequentavam a clínica de odontopediatria da Universidade Cruzeiro do Sul.

Foram incluídas, neste trabalho crianças com a dentição decídua e uma condição sistêmica de saúde normal. Foram excluídas as crianças que apresentaram lesões de cárie dentária e/ou doença periodontal.

Após a aceitação e o preenchimento do termo de consentimento livre e esclarecido pelos responsáveis legais dos menores, as crianças eram encaminhadas à cadeira odontológica para os exames clínicos, realizados por um único examinador estudante de pós-graduação, treinado e calibrado para o diagnóstico de erosão dentária. O examinador foi submetido a duas sessões de 4 horas de treinamento e calibração com exercícios de diagnóstico de erosão dentária, utilizando dez imagens de fotos clínicas e dez dentes extraídos, com diferentes graus de desgaste. As sessões de treinamento foram realizadas por um cirurgião-dentista com experiência no diagnóstico de erosão dentária e o examinador foi submetido ao teste Kappa para confiabilidade intraexaminador.

Também foi aplicado um questionário com questões relativas à dieta alimentar e à qualidade da higiene bucal diária das crianças.

O exame clínico foi feito sob boa iluminação do refletor acoplado ao equipamento, as superfícies dentais foram limpas com uma gaze embebida em água, os dentes foram isolados relativamente com roletes de algodão e secos com o auxílio da seringa de ar. Para melhor visualização, utilizou-se um espelho bucal plano $\mathrm{n}^{\circ} 5$.

As crianças com erosão dentária foram classificadas segundo o índice de $\mathrm{O}^{\prime}$ Brien $^{17}$, devido à sua aplicação e facilidade de uso em diversos trabalhos anteriores em crianças, utilizando escores de um a três para classificar a gravidade da doença.

Após a verificação de presença ou ausência da doença, as crianças foram divididas em dois grupos, sendo vinte para o grupo sem erosão e outras vinte fazendo parte do grupo com erosão.

Os pacientes selecionados para o estudo foram devidamente orientados e remarcados para que em outro dia ocorresse a evidenciação de placa para a classificação do índice de higiene oral simplificado $(\mathrm{IHO}-\mathrm{S})^{18}$. Por meio da quantidade de presença de biofilme, o índice mensura a qualidade da higiene bucal. Para evitar alterações, essa etapa foi padronizada: a realização aconteceu em todos os pacientes dentro do horário das $15 \mathrm{~h}$ às $17 \mathrm{~h}$, as crianças e os pais foram orientados a não fazer a higiene bucal e manter jejum por um período mínimo de 2 horas antes do exame.

$\mathrm{Na}$ segunda etapa, os pacientes eram novamente conduzidos à cadeira odontológica para o exame de IHO-S. Utilizou-se fucsina $3 \%$ como evidenciador do biofilme, que foi aplicado sobre as superfícies dentais com auxílio de cotonete.Os dados foram transcritos em uma planilha.

$\mathrm{O}$ índice de higiene oral simplificado avalia as superfícies vestibular (dentes 16, 11, 26, e 31) e lingual (dentes 36 e 46) - na ausência desses dentes, utilizou-se um dente vizinho como referência -, em que a quantidade de biofilme recebe um código de zero a três em cada superfície. O código zero significa ausência de biofilme; código um, o biofilme não cobre mais do que um terço da superfície examinada; código 2, o biofilme cobre mais de um terço; código 3, o biofilme cobre três terços da superfície dental. A soma dos valores de todas as superfícies avaliadas é dividida pelo número de dentes examinados. A classificação indica um estado bom para valores (com pouco biofilme) de zero a um, regular para os de um a dois e ruim de dois a três ${ }^{18}$.

Os dados foram tabulados no programa Microsof Excel para Windows 7, posteriormente foram elaboradas as tabelas para resumir o conjunto de dados estudados. Quando necessário, foi utilizado o programa Minitab $\mathrm{R}$ versão 14.2 , para o teste de regressão logística de Poisson e dos valores de razão de prevalência (RP), com intervalos de confiança de 95\% (IC 95\%).

\section{Resultados}

Participaram do estudo, quarenta crianças (22 meninos e dezoito meninas), sendo que vinte apresentavam erosão e as outras 20 não apresentavam sinal clínico desse problema (Tabela 1). A idade média (desvio padrão) das crianças com erosão foi de 6,7 (1,3) anos de idade e $7(1,7)$ anos de idade das crianças sem erosão.

Tabela 1 - Número de pacientes (\%) com e sem erosão por gênero

\begin{tabular}{l|r|r|c}
\hline \multicolumn{1}{c|}{ Gênero } & Sem erosão & Com erosão & Total \\
\hline Masculino & $10(45 \%)$ & $12(55 \%)$ & $22(100 \%)$ \\
Feminino & $10(56 \%)$ & $08(44 \%)$ & $18(100 \%)$ \\
Total & $20(50 \%)$ & $20(50 \%)$ & $40(100 \%)$ \\
\hline
\end{tabular}

Fonte: elaboração dos autores com dados da pesquisa. 
O valor de Kappa intraexaminador foi de 0.89 , e 0.84 para o diagnóstico de erosão dentária. A média (desvio padrão) do IHO-S de crianças com erosão foi de $1,20(0,40)$, e das crianças do grupo controle 1,75 $(0,45)$ (Figura 1).

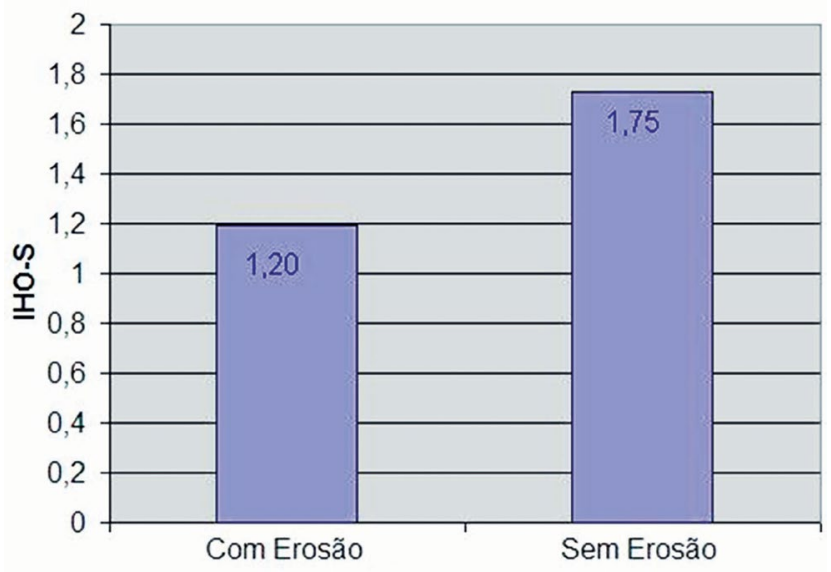

Figura 1 - Quantidade média de biofilme em crianças

A análise de regressão de Poisson, que pode ser observada na Tabela 2, mostrou uma associação entre a quantidade de biofilme dental e a erosão dentária $(\mathrm{p}=0,027)$.

Tabela 2 - Análise de regressão univariada de Poisson com possíveis valores relacionados a crianças com erosão dentária

\begin{tabular}{l|r|r|c}
\hline \multicolumn{1}{c|}{ Variáveis } & RP & \multicolumn{1}{c|}{ IC95\% } & $\begin{array}{l}\text { Valor } \\
\text { de P }\end{array}$ \\
\hline Idade (continua) & 0.634 & $0.350-1.334$ & 0.078 \\
Gênero (ref.: masculino) & 1.00 & 1.00 & 0.591 \\
Feminino & 0.859 & $0.534-1.233$ & \\
IHO-S (continua) & 0.390 & $0.180-0.833$ & 0.027 \\
\hline
\end{tabular}

$\mathrm{RP}$ = razão de prevalência; IC95\% = intervalo de confiança de $95 \%$

Fonte: elaboração dos autores com dados da pesquisa.

A Tabela 3 mostra a distribuição do índice IHO-S e os classifica como bom, regular ou ruim a quantidade de biofilme dental encontrada.

Tabela 3 - Distribuição do índice IHO-S entre os grupos

\begin{tabular}{l|r|r|r}
\multicolumn{1}{c|}{ GRUPO } & Bom & Regular & Ruim \\
\hline Erosão & 3 & 16 & 1 \\
Controle & 1 & 11 & 8 \\
\hline
\end{tabular}

O índice IHO-S classifica como bom os escores de zero a 0,9 quando há pouca presença de biofilme; regular de um a 1,9; ruim de dois a trêsquando há maior quantidade de biofilme acumulada nos dentes.

Fonte: elaborado pelos autores com base nos dados da pesquisa.

\section{Discussão}

O biofilme dental é apontado como sendo um fator possível de proteção contra os desafios ácidos, apontando as regiões da superfície dentária localizadas próximas à margem gengival como não afetadas por agressão ácida por um eventual acúmulo de biofilme ${ }^{11,13}$. Porém, ainda não se sabe a potencial ação protetora do biofilme com a erosão dentária. $\mathrm{O}$ presente estudo quantificou a presença de biofilme dental nas superfícies dos dentes em crianças com erosão dentária, comparando-as com crianças sem a doença, para estabelecer uma possível relação do biofilme como um fator de proteção contra a erosão dentária.

A menor quantidade de biofilme dental em crianças com erosão nos dentes (média de 1,20) comparada com a das crianças que não apresentaram desgastes erosivos nos dentes (média de 1,75), indica realmente haver uma relação de proteção feita pelo biofilme dental, como presumido $(p=0,027)$.

Em um estudo in situ, observou-se a influência do biofilme no esmalte dental. Pacientes usaram aparelhos na boca durante quatorze dias para induzir a formação do biofilme dental, na sequência, os aparelhos foram imersos em refrigerante para produzir a agressão ácida. Pôde-se, então, observar a influência do biofilme no processo de erosão dentária ${ }^{19}$. Os achados desse estudo apontaram para o biofilme como protetor contra a agressão ácida, resultados que condizem com os observados no presente estudo.

Diversas mudanças na cavidade bucal, como alterações no $\mathrm{pH}$ e na temperatura, influenciam na microbiota bucal, podendo modificar a formação do biofilme dental ${ }^{20}$. Ainda não há certeza se os pacientes com erosão nos dentes tinham menor quantidade de biofilme devido à frequente chegada de ácidos à cavidade bucal, que modificaram alguns fatores e influenciaram a formação do biofilme, ou se o desenvolvimento da doença ocorreu pela menor quantidade desse biofilme.

Possivelmente, a escovação dental poderia influenciar na quantidade de biofilme dental presente nas superfícies dos dentes. Para evitar essa possível alteração, as crianças foram orientadas a não fazer a higiene bucal por duas horas antes do exame bucal.

Os meios utilizados para o diagnóstico da erosão dentária e sua classificação têm sido objeto de estudos recentes, pois ainda não existe na literatura um índice universal aceito para a classificação dessas lesões ${ }^{21}$. Em relação ao índice de O'Brien, que surgiu no Reino Unido, é o que tem sido mais utilizado no mundo para estudos e levantamento de prevalência de erosão dentária em crianças. Em alguns desses estudos, houve pequenas modificações, porém, pode ser considerado um índice claro e completo, pois classifica separadamente a profundidade e extensão da lesão erosiva ${ }^{6,22}$.

O índice de erosão dentária utilizado no presente trabalho reflete o diagnóstico diferencial no qual não inclui as outras lesões não cariosas, como abrasão, atrição e abfração, diminuindo a possibilidade de incluir crianças com outros tipos de lesões bucais.

O conhecimento de uma possível relação do biofilme dental com a erosão dentária é de grande importância, pois o processo desse problema de saúde 
bucal, que afeta uma grande parte da população, ainda não está totalmente esclarecido. São necessários novos estudos para que se tenha cada vez mais conhecimento desse problema de saúde bucal que é tão complexo, e se estabelece como uma realidade e preocupação para os cirurgiões-dentistas.

\section{Conclusão}

As crianças com erosão nos dentes, que participaram deste estudo, apresentaram uma quantidade significativamente menor de biofilme em relação às que não tinham erosão. Por isso, conclui-se que, devido à sua propriedade de ação de barreira seletiva, o biofilme pode ser um possível fator protetor contra os ataques ácidos e o desenvolvimento da erosão dentária.

\section{Abstract}

Objective: This study quantified dental biofilm through the Simplified Oral Hygiene Index (OHI-S), by Greene and Vermillion, in children with dental erosion, comparing them with children without this oral health condition. Methodology: The study included 40 children aged 4-9 years, assessed by a single trained examiner according to the $O^{\prime} B r i e n$ index, for the diagnosis of dental erosion, wherein 20 children presented dental erosion and 20 children did not. Thereafter, they received instructions and standardization for the assessment of the OHI-S. Data were analyzed by Poisson's univariate logistic regression. Values of prevalence ratio $(P R)$ and 95\% confidence intervals $(95 \% \mathrm{Cl})$ were calculated. Results: The mean (standard deviation) of the OHI-S of children with erosion was $1.20(0.40)$, which was lower than that of children in the control group, $1.75(0.45)$. The amount of biofilm was associated with the presence of dental erosion (PR 95\% Cl=0.390; 0.180-0.833) and $p$ value $=0.027$. Conclusions: Children with dental erosion showed significantly less biofilm compared to children without this condition. Biofilm may be a potential protection factor against acid attacks and the development of dental erosion.

Keywords: Dental erosion. Dental biofilm. Dental plaque.

\section{Referências}

1. World Health Organization. ICD. International Statistical Classification of Diseases and Related Health Problems, $10^{\text {th }}$ revision, version for 2010 .

2. Black GV. A work on operative dentistry. 2. ed. Chicago: Medico-dental; 1914.

3. Hunter J. The natural history of the human teeth. 2. ed. Londres: [s.n.]; 1728 .

4. Deshpande SD, Hugar SM. Dental erosion in children: An increasing clinical problem. J Ind Soc Prev Dent 2004; 22(3):118-27.

5. Lussi A, Shaffner M, Jaeggi T. Dental erosion - diagnosis and prevention in children and adults. Int Dent J 2007; 57:385-98.
6. Murakami C, Oliveira LB, Sheiham A, Corrêa MSNP, Haddad AE, Bönecker M. Risk indicators for erosive tooth wear in brazilian preschool children. Caries Res 2011; 45(2):121-9.

7. Zero DT, Lussi A. Erosion: chemical and biological factors of importance to the dental practitioner. Int Dent J 2005; 55(4):285-90.

8. Huysmans MCDNJM, Chew HP, Ellwood RP. Clinical studies of dental erosion and erosive wear. Caries Res 2011; 45(1):60-8.

9. Wang X, Lussi A. Assessment and management of dental erosion. Dent Clin North Am 2010; 54(3):565-78.

10. Corrêa MSNP, Corrêa FNP, Corrêa JPNP, Murakami C, Mendes FM. Prevalence and associated factors of dental erosion in children and adolescents of a private dental practice. Int J Paediatr Dent 2011; 1-8.

11. Ganss C. How valid are current diagnostic criteria for dental erosion? Clin Oral Invest 2008; 12:41-9.

12. Bartlett DW. The role of erosion in tooth wear: Aetiology, prevention and management. Int Dent J 2005; 55:277-84.

13. Lussi A, Schlueter N, Rakhmatullina E, Ganss C. Dental erosion - An overview with emphasis on chemical and histopathological aspects. Caries Res 2011; 45(1):2-12.

14. Nunn JN, Gordon PH, Morris AJ, Pine CM, Walker A. Dental erosion: changing prevalence? A review of British national childrens' surverys. Int J Paed Dent 2003; 13:98-105.

15. Young A, Tenuta LMA. Initial erosion models. Caries Res 2011; 45(1):33-42.

16. Simain F, Rompen E, Heinen E. Dental biofilms. Rev Med Liege 2010; 65(10):569-73.

17. O'Brien M. Children's dental health in the United Kingdom, 1993. Office of population censuses and surveys. London: Her Majesty's Stationery Office; 1994. 74-76.

18. Greene JC, Vermillion JR. The simplified oral hygiene index. J Am Dent Assoc 1964; 68:7-13.

19. Honório HM, Rios D, Santos CF, Buzalaf MA, Machado MA. Influence of dental plaque on human enamel erosion: in situ / ex vivo study. Oral Health Prev Dent 2010; 8(2):179-84.

20. Marsh PD, Devine DA. How is the development of dental biofilms influenced by the host? J Clin Periodontol 2011; 38(11):28-35.

21. Harding MA, Whelton H, O’Mullane DM, Cronin M. Dental erosion in 5-years-old Irish school children and associated factors: a pilot study. Community Dent Health 2003; 20(3):165-70.

22. Gurgel CV, Rios D, Buzalaf MA, Silva SM, Araújo JJ, Pauletto AR, et al. Dental erosion in a group of 12 - and 16 - year -old Brazilian schoolchildren. Pediatr Dent 2011; 33(1):23-8.

\section{Endereço para correspondência:}

Caleb Moreira Shitsuka

Faculdade de Odontologia da Universidade de São Paulo Av. Lineu Prestes, 2.227

05508-000 São Paulo, São Paulo, Brasil

Telefone: 1130917835 / 30917854

E-mail: caleb@usp.br

Recebido: 16/12/14. Aceito: 09/09/15. 